\title{
High-throughput exposure modeling to support prioritization of chemicals in personal care products
}

Susan A. Csiszar ${ }^{* a, b}$, Alexi S. Ernstoff ${ }^{\mathrm{c}}$, Peter Fantke ${ }^{\mathrm{c}}$, David E. Meyer ${ }^{\mathrm{b}}$, Olivier Jolliet ${ }^{\mathrm{d}}$

${ }^{a}$ Oak Ridge Institute for Science and Education (ORISE) Research Participation Program, Oak Ridge, TN 37831-0117, USA

${ }^{\mathrm{b}}$ Office of Research and Development, National Risk Management Research Laboratory, U.S. Environmental Protection Agency (EPA), Cincinnati 45268, OH, USA

${ }^{c}$ Quantitative Sustainability Assessment Division, Department of Management Engineering, Technical University of Denmark, Kgs. Lyngby, Denmark

${ }^{\mathrm{d}}$ Environmental Health Sciences, School of Public Health, University of Michigan, Ann Arbor, MI, United States

"Corresponding author: csiszar.susan@epa.gov, 1-513-569-7324

\begin{abstract}
We demonstrate the application of a high-throughput modeling framework to estimate exposure to chemicals used in personal care products (PCPs). As a basis for estimating exposure, we use the product intake fraction (PiF), defined as the mass of chemical taken by an individual or population per mass of a given chemical used in a product. We calculated use- and disposal- stage PiFs for 518 chemicals for five PCP archetypes. Across all product archetypes the use- and disposal- stage PiFs ranged from $10^{-5}$ to 1 and 0 to $10^{-3}$, respectively. There is a distinction between the use-stage PiF for leave-on and wash-off products which had median PiFs of 0.5 and 0.02 across the 518 chemicals, respectively. The PiF is a function of product characteristics and physico-chemical properties and is maximized when skin permeability is high and volatility is low such that there is no competition between skin and air losses from the applied product. PCP chemical contents (i.e. concentrations) were available for 325 chemicals and were combined with PCP usage characteristics and PiF
\end{abstract}


yielding intakes summed across a demonstrative set of products ranging from $10^{-8}$ to 30 $\mathrm{mg} / \mathrm{kg} / \mathrm{d}$, with a median of $0.1 \mathrm{mg} / \mathrm{kg} / \mathrm{d}$. The highest intakes were associated with body lotion. Bioactive doses derived from high-throughput in vitro toxicity data were combined with the estimated PiFs to demonstrate an approach to estimate bioactive equivalent chemical content and to screen chemicals for risk.

Keywords exposure modeling; personal care products; mass balance modeling; product intake fraction; risk screening; high-throughput

\section{Introduction}

There are potentially thousands of chemicals used in personal care products (PCP) and cosmetics (Egeghy et al., 2011) and estimating exposure to all these chemicals is not possible based on empirical techniques alone. Biomonitoring data, for example, are only available for a subset of compounds through programs such as NHANES (CDC, 2009) in the United States. Computational exposure models can be used to estimate chemical intake due to the use of PCPs based on physico-chemical properties, product composition, and product usage characteristics for chemicals in the absense of emperical data.

There are several examples of high-throughput (HT) exposure models for chemical prioritization and screening (Wambaugh et al., 2013; Isaacs et al., 2014; Shin et al., 2015).These HT exposure estimates are being combined with HT screening toxicity data, for example in vitro bioactivities from the U.S. EPA ToxCast program (Kavlock et al., 2012), to screen and prioritize chemicals for risk (Cohen Hubal et al., 2010; Shin et al., 2015; Wetmore et al., 2015). These efforts have highlighted the need to better understand chemical exposure from consumer products (Wambaugh et al., 2013; 2014; Shin et al., 2015). PCPs are a 
product class for which emperical studies have found that usage is correlated with chemical exposures (Sandanger et al., 2011; Parlett et al., 2013).

Jolliet et al. (2015) have proposed the product intake fraction (PiF) metric to quantity the amount of chemical taken in by the exposed population per mass of chemical used in a consumer product. Shin et al. (2015) combined the PiF with chemical production volumes (PVs) to estimate dermal exposure to chemicals in PCPs using body lotion as a sentinel product. PVs, however, do not inform what proportion of the PV is used in any given product type or process nor on how many members of the population use a given chemical (Nazaroff et al., 2012; Shin et al., 2015). Dudzina et al. (2015) modeled route specific exposure fractions to PCP chemicals, however, this method was applied to a single chemical and has yet to be applied to several PCP chemicals at once. Recently, Csiszar et al. (2016) applied the $\mathrm{PiF}$ to estimate dermal and inhalation exposure to parabens in several PCP types using chemical fractional content (i.e., concentrations) with exposure estimates which compared well to biomonitoring data. The PiF can also be used to estimate environmentally mediated exposures to post-use emissions (referred to as disposal-stage) (Jolliet et al., 2015) although this has also yet to be performed in an HT study.

In this paper, we combine PiFs with product usage and fractional chemical content data to develop an HT exposure and risk screening approach to estimate use- and disposal-stage exposures to hundreds of PCP-chemical combinations. The goals of this study are to: (i) demonstrate the use of PiF to estimate exposure to hundreds of PCP chemicals, (ii) understand chemical and product specific factors affecting PiF, and (iii) within a risk screening context, use bioactive doses derived from in vitro screening toxicity data (Wetmore 
et al., 2012; 2015) to demonstrate how bioactive chemical contents can be back-calculated and compared with actual chemical contents being used in PCPs (Goldsmith et al., 2014).

\section{Methods}

\subsection{Product intake fraction for PCPs}

We considered four exposure pathways for calculating PiF due to the application of PCPs to the skin. Dermal aqueous uptake $\left(P i F^{\text {derm, aq }}\right)$, inhalation $\left(P i F^{u s e, i n h}\right)$, and dermal gaseous uptake $\left(P i F^{d e r m, g}\right)$, comprise the use-stage $\mathrm{PiF}$ and can be summed as $P i F^{u s e, t o t}$. The fourth pathway is an aggregated intake via outdoor environmental exposure pathways $\left(P i F^{d i s p}\right)$. The sum of all four pathways is $P i F^{t o t}$. Following Csiszar et al. (2016) and Ernstoff et al. (2016) for a product that is applied to skin, a mass-balance can be analytically solved to calculate the fraction of chemical lost from the product via transfer into the skin or volatilization to air $\left(f^{\text {volat }}\right)$. The fraction that is transferred to skin from the product is also the fraction of dermal aqueous uptake, i.e. $P i F^{d e r m, a q}$ and the solution is

$$
P i F^{d e r m, a q}=\frac{k_{p s}}{k_{p s}+k_{p a}}\left(1-e^{-\left(k_{p s}+k_{p a}\right) t}\right) ; f^{v o l a t}=\frac{k_{p a}}{k_{p s}+k_{p a}}\left(1-e^{-\left(k_{p s}+k_{p a}\right) t}\right)
$$

where $k_{p s}$ and $k_{p a}$ are the product-to-skin and product-to-air transfer rates $\left(\mathrm{h}^{-1}\right)$, respectively, and $t$ is the application duration (h) (see Supplementary Information (SI) for more information). The $k_{p s}$ is a function of the aqueous skin permeation coefficient, $K_{p}^{a q}(\mathrm{~m} / \mathrm{h})$ which was calculated using the relationship of ten Berge (2009) based on molecular weight $(M W)$ and octanol-water partition coefficient $\left(K_{o w}\right)$. The $k_{p a}$ is a function of air-water mass transfer coefficient and depends on the air-water partition coefficient, $K_{a w}$. The fraction of chemical that volatilizes to air becomes available for inhalation and gaseous dermal uptake and transfer to outdoor air. We assumed that chemicals volatilize to a near-person area of 1 
$\mathrm{m}^{3}$ (Isaacs et al., 2014) and then transfer to a larger well-mixed indoor air compartment. After the exposure duration, $t$, the fraction of chemical remaining in the applied product (i.e., on the skin surface) is assumed to be washed down the drain to a waste water treatment plant (WWTP) resulting in subsequent fractions emitted to air, water, and soil. To estimate $P i F^{d i s p}$ we multiplied the emission fractions to WWTPs and outdoor air with their respective outdoor mediated intake fractions (mass of chemical taken in per mass of chemical emitted) for release to air, water, and soil calculated using the USEtox model (Rosenbaum et al., 2008). These processes are summarized in Figure S1 and equations are listed in Tables S1 and S2 in the SI.

Product and exposure characteristics (i.e., irrespective of chemical properties) which determine the PiF include product use duration, product thickness, room ventilation rates, inhalation rates, and surface area of skin in contact with air (Csiszar et al., 2016). Product characteristics were grouped into five different product archetypes: 'leave-on'; 'leave-on, spray'; 'shampoo'; 'body wash'; 'face wash'; with distinguishing parameters summarized in Table S3 and based on Csiszar et al. (2016). Incidental ingestion, for example, from hand-tomouth contact can also be an exposure pathway for PCP chemicals. We did not model this pathway for most products since we assumed that it was negligible for wash-off products and small compared to dermal uptake for leave-on products. This pathway is likely most important for products with lip application. To address this, we included a $1 \%$ ingestion of product irrespective of chemical properties (Isaacs et al., 2014) and the remaining 99\% treated as the 'leave-on' archetype. This method or more elaborate hand to mouth models based on contact frequency could also be used to include incidental ingestion for other 
product types, especially for chemicals which have small PiFs for the other considered pathways.

\subsection{Intake and risk screening}

The PiF can be combined with chemical content and product use information to estimate chemical intakes following Csiszar et al. (2016). The intake $(\mathrm{mg} / \mathrm{kg} / \mathrm{d})$ of a chemical, $c$, due to the use of a single PCP, $p$, can be calculated as

$$
I_{c, p}=\frac{P_{p} f_{c, p} P i F_{c, p}^{t o t}}{B W}
$$

where $P_{p}$ is the mass of product $p$ used per person per day $(\mathrm{mg} / \mathrm{d}), f_{c, p}$ is the fractional chemical content in a given product, and $B W(\mathrm{~kg})$ is body weight. In this paper, aggregate PCP exposure to one chemical refers to the sum of intakes across the eleven products types that chemical was found to be used in (referred to as relevant product types) and calculated as

$$
I_{c}=\frac{1}{B W} \sum_{p} P_{p} f_{c, p} P i F_{c, p}^{t o t}
$$

If mean population level aggregate intakes are required, intakes can be adjusted for the fraction of the population using a given PCP and the prevalence of a chemical in a given PCP as suggested by Cowan-Ellsberry and Robison (2009). In this study, the occurrence of a chemical in a given product type was accounted for by only considering product-chemical combinations derived from the U.S. EPA Consumer Product Chemical Profile database (CPCPdb) (Goldsmith et al., 2014). To obtain a range of both single product and aggregate intakes we used mean, minimum, and maximum values of $f_{c, p}$ across a given product type and the mean, $10^{\text {th }}$, and $95^{\text {th }}$ percentiles of $P_{p}$ (Loretz et al., 2005; 2006; 2008) and refer to these as mean, low, and high intakes, respectively. 
We back-calculated bioactive equivalent chemical contents, BEC, that is, chemicalspecific PCP content which would yield a biological response based on PiF-estimated exposures. These can be based on toxicity metrics, such as minimum oral equivalency doses (OEDs) derived from in vitro screening toxicity data (Wetmore et al., 2012; 2015) which have been used previously to screen chemicals for risk (Shin et al., 2015). The intake equation (Eq. 3) can be re-arranged to solve for fractional $B E C_{c}$, and by replacing intake, $I_{c}$ with an $O E D_{c}(\mathrm{mg} / \mathrm{kg} / \mathrm{d})$ as

$$
B E C_{c}=\frac{O E D_{c} \times B W}{\sum_{p} P_{p} P i F_{c, p}^{t o t}} .
$$

This $B E C c$ represents the bioactive concentration assuming that intake is aggregated across PCPs and thus represents an average chemical content within each PCP considered in the sum and is not product specific. We considered two distinct scenarios to calculate a range in $B E C_{c}$. For a given chemical, we assumed that an individual used all products in which the chemical was found to occur (i.e. relevant products) and a second hypothetical situation where a user is exposed to this chemical via all 11 considered PCPs. Additionally, we used the $95^{\text {th }}$ percentile $P_{p}$ from Loretz et al. $(2005,2006,2008)$, to model a "high-use" individual using all PCPs in high amounts. To screen for potential risks, BECs were compared toreported chemical contents.(Goldsmith et al., 2014)

\subsection{Identifying Chemicals used in PCPs}

Chemicals used in PCPs were identified using the U.S. EPA CPCPdb (Goldsmith et al., 2014) which provides product specific chemical contents. The products in the CPCPdb were mapped to product categories used in the U.S. EPA SHEDS-HT consumer product exposure model (Isaacs et al. 2014, personal communication) and these categories were used to 
identify chemicals and associated contents used in PCPs. The chemical list was additionally filtered based on availability of chemical properties in EPISuite (U.S. EPA, 2012) and falling within the applicability range of the $K_{p}^{a q}$ relationship (log $K_{\text {ow: }}-4$ to 6; $M W: 10$ to 1000 $\mathrm{g} / \mathrm{mol}$ ) (ten Berge, 2009) yielding a total of 518 chemicals. This list of chemicals was used to calculate PiFs for a range of chemicals that could be used in PCPs.

To demonstrate chemical intake calculations, the amount of PCP used is needed as well as information on the chemical concentrations in those products (Equation 2). For these demonstrative calculations, we chose a subset of eleven commonly used PCPs (body lotion, body wash, conditioner, deodorant, eye shadow, face cleanser, face cream, foundation, lipstick, perfume, shampoo) with readily available product mass usage information for the US female population (Loretz et al., 2005; 2006; 2008) (Table S5), a population group with wide PCP usage (Wu et al., 2010; Biesterbos et al., 2013). The SHEDS-HT product categories (Isaacs et al., 2014) were mapped to these eleven categories and were assigned each of the eleven product categories to one of the five PiF archetypes (Table S3) to calculate product specific PiFs. Several product-chemical combinations did not have specific fractional chemical contents thus limiting the number of chemicals with chemical content information to 325 (i.e. these chemicals had at least one product with a reported concentration). These chemicals were used to demonstrate intake estimates. Of these 325 chemicals, in some cases, a chemical was identified as occurring in a specific product type without concentration information for that specific product type; in these cases we used the average chemical content for that chemical across the other PCP types (Table S6).

\section{Results}




\subsection{PiFs for PCP chemicals}

We calculated PiFs for the 518 chemicals for all five PCP archetypes. Across all archetypes the use- and disposal- stage PiFs ranged from $10^{-5}$ to 1 and 0 to $10^{-3}$, respectively. The wash-off archetypes had the largest $P i F^{\text {disp }}$ values and for most chemicals the use-stage $\mathrm{PiF}$ was several orders of magnitude larger than the disposal-phase PiF, however, $\sim 35 \%$ of chemicals had $P i F^{\text {disp }}$ values within an order of magnitude of $P i F^{\text {use,tot }}$ (for the shampoo archetype). For these chemicals, however, the largest $P i F^{t o t}$ was 0.006 indicating that when $P i F^{u s e}$ and $P i F^{d i s p}$ have similar values, the total $\mathrm{PiF}$ is relatively small compared to other chemicals which can have $P i F^{t o t}$ up to unity. The $P i F^{u s e}$ for the leave-on product archetype was at least an order of magnitude larger than $P i F^{\text {disp }}$ for all chemicals. It follows that individual exposure to most PCP chemicals is generally dominated by the use-stage (especially for leave-on products) rather than the disposal stage once a chemical has been emitted outdoors. This is supported by studies which have found correlations between PCP usage and measured levels of chemicals such as phthalates and parabens in urine (Sandanger et al., 2011; Parlett et al., 2013; Harley et al., 2016).

The cumulative distributions of the use-stage PiF dermal and inhalation routes for the 518 chemicals can be found in Figure 1. The dermal uptake route dominates the PiF ${ }^{\text {usetot }}$ for all archetypes. The maximum PiF $F^{\text {use inh }}$ is bounded by the inhalation intake fraction (iF) estimated approximately as the ratio of the inhalation rate $(I R)$ to the air ventilation rate $(V R)$ (see discussion in the SI) (Figure S2). While there is considerable uncertainty in the inhalation $\mathrm{iF}$ which was bounded by approximately 0.04 , it is similar to the indoor $\mathrm{iF}$ of 0.01 as estimated by Hellweg et al. (2009). Combining the inhalation intake fraction and the fraction volatilized results in a limited contribution of $P i F^{u s e, i n h}$ to $P i F^{u s e, \text { tot. }}$ 
There is a clear distinction between the PiF ${ }^{\text {use,tot }}$ for leave-on versus wash-off products with medians across the 518 chemicals of 0.5 and 0.02 for the 'leave-on' and 'shampoo' archetypes, respectively. The 'leave-on, spray' category yielded a similar PiF ${ }^{\text {use,tot }}$ cumulative distribution to the 'leave-on' products as both categories had median PiF ${ }^{\text {use,tot }}$ of 0.5 , resulting from the limited contribution of PiF ${ }^{u s e, i n h}$ described above. Of the three wash-off archetypes the 'body wash' category yielded the highest $P i F^{u s e, t o t}$ values due to the larger surface area of application (Eq. 1, Table S3) (Csiszar et al., 2016).
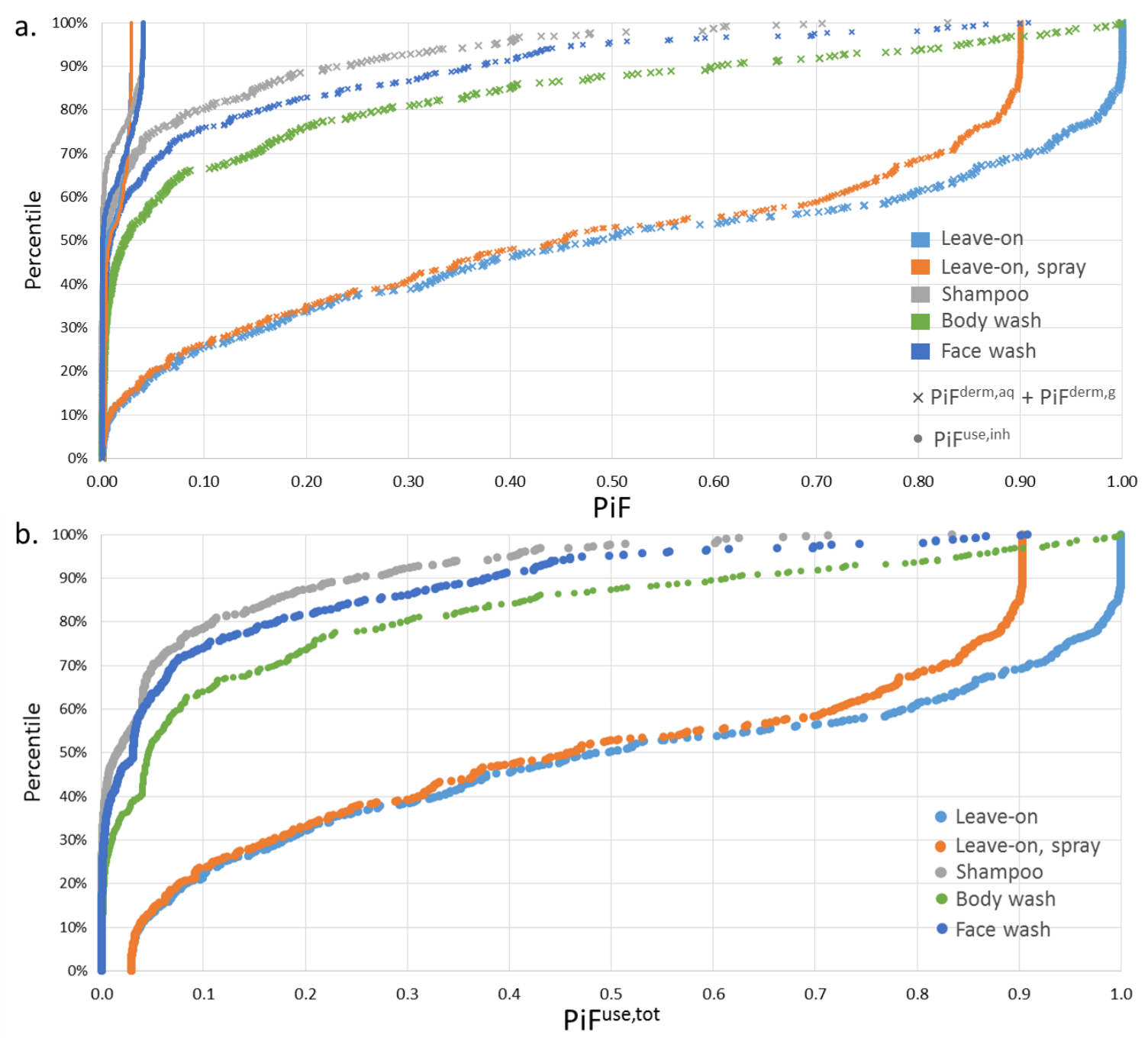
Figure 1: Cumulative distributions of the 518 PCP chemicals for the five PCP archetypes for the a) dermal PiF $\left(P i F^{d e r m, a q}+P i F^{d e r m, g}\right)$ and $P i F^{u s e, i n h}$, and b) PiFuse,tot.

\subsection{PiF and chemical properties}

In this model, the PiF $F^{u s e, t o t}$ depends on three physico-chemical properties: $K_{a w}$, and $K_{\text {ow }}$ and molecular weight $(M W)$, primarily via the $K_{p}^{a q}$ relationship. To understand how properties mediate PCP exposure during use we plotted the use-stage PiF as a function of log $K_{p}^{a q}$ (which is a function of $\mathrm{K}_{\mathrm{ow}}$ and $\left.M W\right)$ and $\log K_{a w}$ at the median $M W(195 \mathrm{~g} / \mathrm{mol})$ for the two most distinct archetypes of 'leave-on' and 'shampoo'. The PCP chemicals in this paper cover a wide range of properties (log $\mathrm{K}_{\mathrm{aw}}$ : -42 to $2.6 ; \log K_{p}^{a q}(\mathrm{~cm} / \mathrm{h})-5.4$ to 0.6) (Figure 2).

Equation 1 and Figure 2 demonstrate $P i F^{d e r m, a q}$ is maximized when skin permeability is high and volatility is low such that there is no competition between skin and air losses from the applied product. For the 'leave-on' archetype, we can distinguish three main zones: at low $\mathrm{K}_{\mathrm{aw}}$ (i.e., $<10^{-8}, \sim 40 \%$ of the considered chemicals) where volatilization of the applied product is small compared to dermal uptake and wash-off. For most chemicals, PiF ${ }^{d e r m, a q}$ and therefore $P i F^{u s e, t o t}$ are high (i.e. close to 1 ) and it is only for very low $K_{p}^{a q}$ (i.e. $<\sim 10^{-5} \mathrm{~cm} / \mathrm{h}$ ) and for a few of the considered chemicals that PiFuse,tot was less than 0.5.

In the intermediary zone, $\left(-8 \leq \log \mathrm{K}_{\mathrm{aw}} \leq-3, \sim 40 \%\right.$ of the considered chemicals $)$ volatilization competes with dermal uptake, which is reflected by diagonal projected in the $\mathrm{K}_{\mathrm{aw}} / K_{p}^{a q}$ space. (Figure 2a).This demonstrates the importance of using a mass balance model that accounts for the competition between the different exposure routes. Overall the PiF use,tot decreases with increasing $\mathrm{K}_{\mathrm{aw}}$ as $P i F^{u s e, i n h}$ is limited by the indoor intake fraction, whereas PiF ${ }^{\text {derm,aq }}$ is closer to 1 at higher $K_{p}^{a q}$. For a subset of properties, the $P i F^{d e r m, g}$ can be larger 
than PiF ${ }^{\text {use,inh }}$ for chemicals which readily volatilize from an aqueous solution, however, also partition favorably from air to skin. This has also been found by Weschler and Nazaroff (2014) for several chemicals.

At high $\mathrm{K}_{\mathrm{aw}}$ (i.e. $>10^{-3}, \sim 20 \%$ of the considered chemicals), volatilization substantially contributes to the removal of chemical applied to skin and limits the PiF $F^{d e r m, a q}$, even at high $K_{p}^{a q}$. For the chemicals considered in this study, $P i F^{\text {use,tot }}$ was bound $\sim 0.5$ in this zone.

Overall, for the 'leave-on' archetype, this leads to $\sim 50 \%$ of chemical with PiF use,tot greater than or equal to 0.5 dominated by dermal uptake, and $\sim 10 \%$ with PiF use,tot less than $\sim 0.04$ dominated by inhalation. The remaining $\sim 40 \%$ are intermediate chemicals for which both volatilization and dermal uptake are of similar importance in terms of ingredient removal and exposure.

The 'shampoo' archetype follows similar patterns, however, generally with a substantially higher fraction washed-off due to the shorter exposure time (i.e. on the order of minutes compared to hours), which limits the dermal uptake, especially at low $K_{p}^{a q}$. Reducing the exposure duration also reduces the exposure potential for most property combinations. However, for chemicals with relatively fast skin permeability values (e.g. $\sim 1 \mathrm{~cm} / \mathrm{h}$ ), dermal uptake reaches plateau often within just a few minutes. Thus, reducing the exposure duration for high skin permeability compounds does not generally decrease the PiF use,tot. Of the 518 chemicals studied, only a small subset fell into this category (Figure 2). Nevertheless, of these few chemicals some are matched to uses in over 100 PCPs suggesting their use can be common. Additionally, for chemicals with relatively low 'leave-on' PiF' ${ }^{\text {use,tot }}$ (e.g. $<0.1$ ), 
reducing exposure duration can also yield PiFs within a factor of one between the exposure scenarios; this also occurred for a subset of chemicals as shown on Figure 2."

$\log \mathrm{PiF}^{\text {derm, aq }}$

a. leave-on

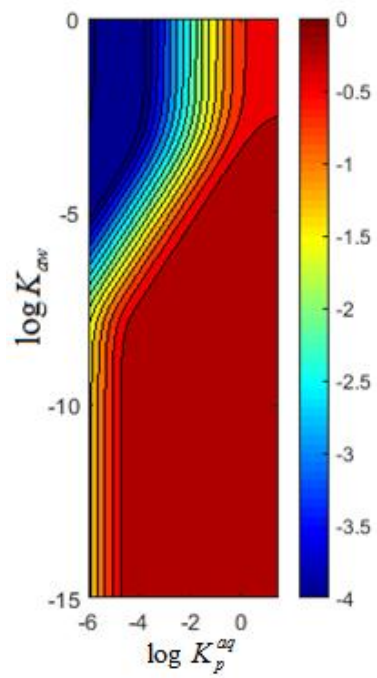

b. shampoo

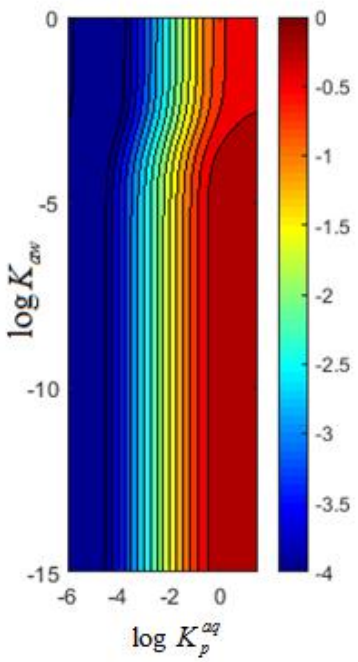

$\log$ PiFuse,inh
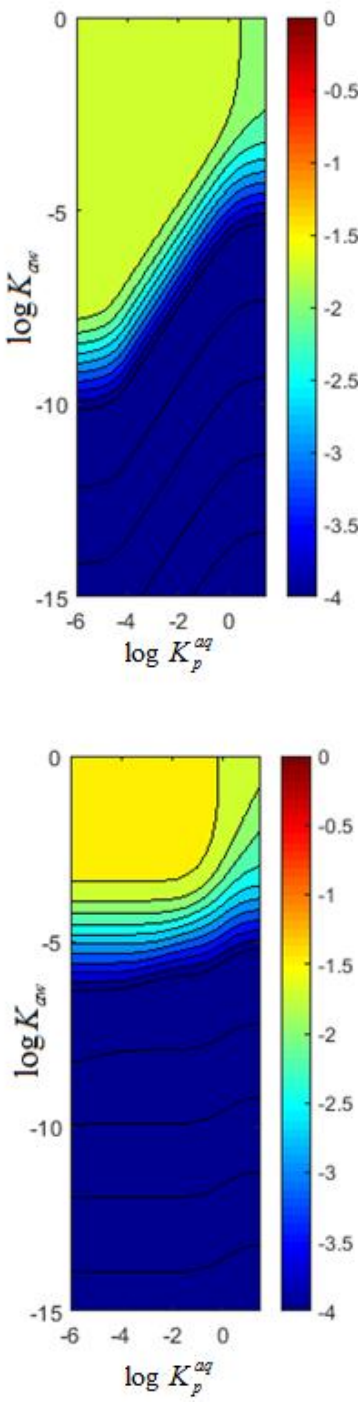

$\log \mathrm{PiF}^{\text {derm,g }}$
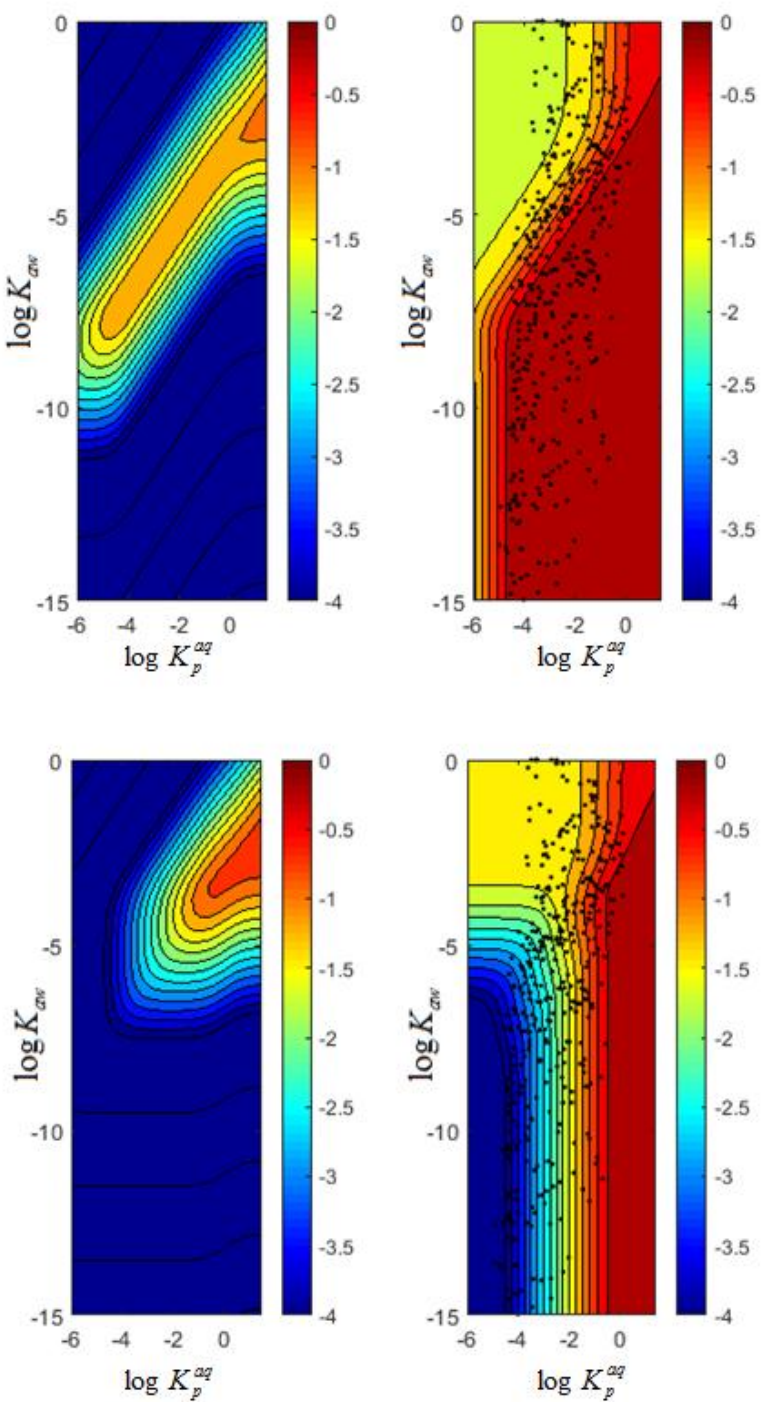

$\log$ PiFuse,tot

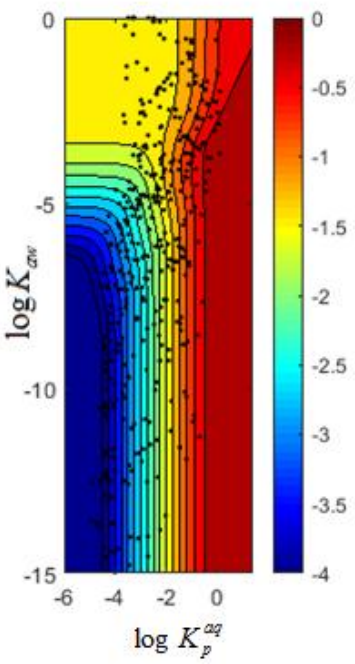

Figure 2:. The use-stage $\log _{10} \mathrm{PiFs}$ as a function of $\log _{10} \mathrm{~K}_{\mathrm{ow}}$ and $\log _{10} K_{p}^{a q}(\mathrm{~cm} / \mathrm{h})$ for a) 'leave-on' and b) 'shampoo' product archetypes at the median molecular weights of the PCP chemicals. The plot of $\log P i F^{u s e, t o t}$ includes the $\mathrm{K}_{\mathrm{aw}}$ and $K_{p}^{a q}$ values from the PCP chemicals in this study as black dots.

Notes: The molecular weights of the chemicals also vary, such $P i F^{u s e, t o t}$ values on this plot are approximate for the actual chemicals. Some chemicals had properties which did not fall within the values of these demonstrative plots and were thus not included in the figure. 


\subsection{Mass applied and chemical intakes}

Across the 325 chemicals, mean PCP content ranged from $10^{-6}-55 \%$ (low: $10^{-6}-$ 51\%, high: $10^{-6}-100 \%$ ), yielding masses of chemical applied ranging from $10^{-8}-250$ $\mathrm{mg} / \mathrm{kg} / \mathrm{d}$ (low: $10^{-9}-66 \mathrm{mg} / \mathrm{kg} / \mathrm{d}$; high: $10^{-8}-680 \mathrm{mg} / \mathrm{kg} / \mathrm{d}$ ) aggregated over the relevant products. The aggregated mass applied across the considered products was dominated by the wash-off products conditioner, body wash, and shampoo and the leave-on product body lotion (Figure 3a). Applying PiF $F^{\text {tot }}$ to the mass of chemical applied (to each product) reduced the intake aggregated across products by two orders of magnitude on average (range: factor

of 1 to $10^{4}$ ). The mean chemical intake aggregated across the product types ranged from $10^{-8}$ - $30 \mathrm{mg} / \mathrm{kg} / \mathrm{d}$ (low: $10^{-9}-8 \mathrm{mg} / \mathrm{kg} / \mathrm{d}$; high: $10^{-8}-85 \mathrm{mg} / \mathrm{kg} / \mathrm{d}$ ) across the 325 chemicals, with the highest intakes associated with body lotion (Figure 3b).

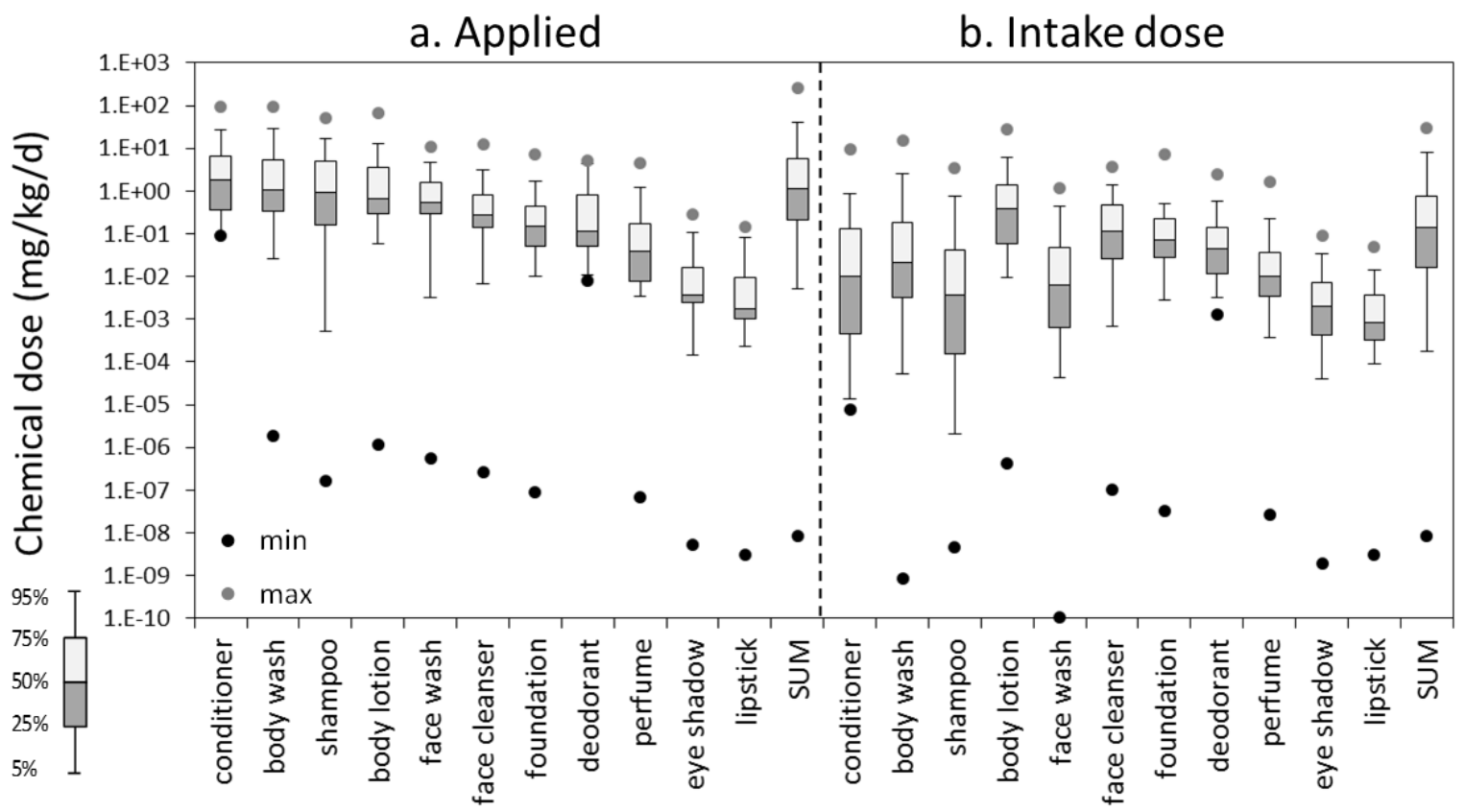

Figure 3: Ranges in a) mean mass of chemical applied, i.e. mean product mass applied multiplied with mean chemical content, and b) intake doses after applying the PiFtot across 
the 325 chemicals with intake estimates. The "SUM" column refers to doses aggregated over the eleven products for each chemical.

Fourteen of the PCP chemicals assessed here also had screening intakes estimated by Shin et al. (2015) using PVs and the PiF $F^{d e r m, a q}$ assuming a body lotion archetype. We verified that these chemicals are used in PCPs by checking that they appear in other databases (U.S. NIH, 2015) (Dionisio et al., 2015) as having PCP use. After this, one chemical was removed from the list resulting in 13 chemicals for the analysis. For seven of the chemicals, the mean mass of chemical applied and estimated intakes (aggregated across products) in this paper were larger than those using the methods of Shin et al. (2015) (Figure S3). For three of these chemicals (ethyl-, propyl, and butyl-paraben), there was no overlap between estimates in this paper and those using the methods of the Shin et al (2015) (Figure S3b). Csiszar et al. (2016) estimated intake distributions for parabens, also using product content as input (rather than PVs), and found that the estimated exposures agreed well with biomonitoring data. The mean paraben intakes of Csiszar et al. (2016) were also higher than those estimated by Shin et al. (2015) (Figure S3b) indicating that using PVs may in certain cases underestimate mass applied and therefore chemical exposure due to PCP usage. This may be especially relevant for chemicals such as parabens that are mostly used in PCPs, and when assuming that an individual is exposed via all considered PCPs.

\subsection{Bioactive equivalent chemical contents and risk screening}

Of the chemicals with estimated intakes there were 18 which also have OEDs, derived from in vitro toxicity data (Wetmore et al., 2012; Shin et al., 2015; Wetmore et al., 2015). We removed one chemical from this list (resulting in 17 chemicals) as we verified that these chemicals are used in PCPs as described for the comparison to Shin et al. (2015). For these chemicals, we back-calculated bioactive equivalent chemical contents (BECs) by 
assuming that a given chemical occurs in either each of the 11 product types or only in the relevant product types (i.e. those that were found to contain a given chemical derived from the CPCPdb). This yielded ranges in back-calculated BECs of $\sim 10^{-4}-16 \%$ and $\sim 10^{-3}-$ $100 \%$, respectively. For 6 of the 17 chemicals, the mean chemical content derived from the CPCPdb was higher than the back-calculated BEC for the relevant product types and four more were within a factor of ten of the mean chemical content (Figure 4). Assuming that a given chemical occurred in all versus relevant product types decreased the back-calculated BECs by up to two orders of magnitude. Comparing BECs calculated assuming chemical occurrence in all product types, to the maximum chemical contents derived from the CPCPdb indicated that all but one of the chemicals were within a factor of ten of the BEC. While this analysis was only conducted for a small subset of chemicals it demonstrates how the PiF can be used with bioactive doses to calculate bioactive chemical contents and to screen chemicals for risk. 


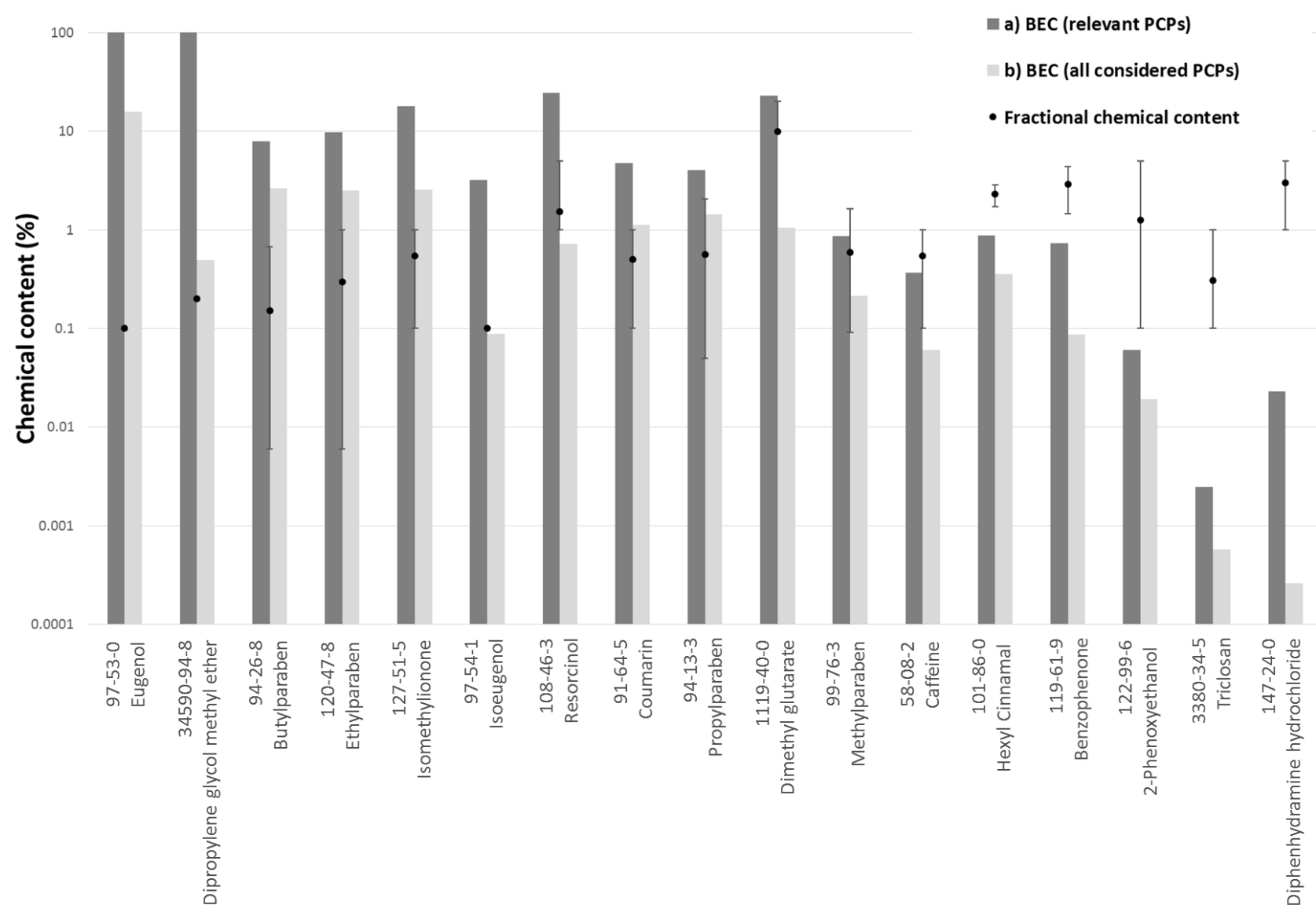

Figure 4: Back-calculated bioactive equivalent chemical contents (BECs) assuming that a given chemical is used in a) only relevant PCPs (i.e. those PCPs in which the chemical appeared, derived from Goldsmith et al. (2014)) and b) all 11 considered PCPs; and comparison with c) mean chemical content found in the considered PCPs derived from Goldsmith et al. (2014). The error bars represent the mean of the low and high content across the considered PCP types; some chemicals did not have ranges available and thus do not have error bars.

\section{Discussion}

We demonstrated how the PiF can be used to rapidly estimate exposure potentials for chemicals in PCPs. The PiF models used in this study allowed estimation of multi-pathway exposures which accounted for the transfer fractions, for example, from skin to air. This method also allows for estimating exposure due to outdoor releases associated with product use, thereby coupling near-field chemical release fate and exposure with far-field ones. The 
plots of $\mathrm{PiF}$ as a function of chemical properties give insight into properties which mediate exposure (Figure 2). For example, Figure 2a demonstrates that for low volatility chemicals (i.e. $K_{a w}$ less than $10^{-8}$ ), exposure potential is driven by the skin permeability of the chemical; and for leave-on products, low values of $K_{p}^{a q}$ (e.g. $<\sim 10^{-5} \mathrm{~cm} / \mathrm{h}$ ) were needed to yield $P i F^{u s e, t o t}$ values less than 0.5 . As $K_{a w}$ increases, dermal permeability and volatility become competing processes influencing exposure potentials. An understanding of the effects of chemical properties on the PiF could potentially inform the design or choice of chemicals in PCPs to minimize exposure potential.

There was a clear distinction between the use-stage PiFs for leave-on compared to wash-off products (Figure 1), suggesting that for HT purposes, it may only be necessary to distinguish between either a 'leave-on' or 'wash-off' product when calculating a PiF rather than further distinguishing between product use scenarios. Applying the PiF to hundreds of PCP chemicals indicated that the use-stage PiF dominates chemical intake for most productchemical combinations, but not necessarily for all. Furthermore, there are members of the population who are not exposed to a given chemical in the use-stage (i.e. they do not use PCPs containing specific chemicals) thus their exposure would only occur from outdoor chemical releases. This indicates that intakes due to outdoor releases should also be included in exposure assessments, especially for wash-off products with the largest $P i F^{d i s p}$ values.

Chemical contents within a given PCP type varied over several orders of magnitude yielding a large variation in applied doses, however, this variability could be lessened when comparing across chemicals with similar function as they may have similar chemical properties (Egeghy et al., 2015). For leave-on products there is a much smaller variation in $\mathrm{PiF}$ across products than there is in chemical content, however, applying the PiF can still 
change estimated intakes by an order of magnitude. This suggests that for leave-on products the PiF may have a smaller contribution to overall exposure than product chemical content data. For rinse-off products, on the other hand, the variation in PiF and content are of similar orders of magnitude indicating that both these terms can contribute substantially to final estimated intake doses.

Comparing our results to those of Shin et al. (2015) indicates that using PVs may not necessarily provide the most conservative screening level exposures, highlighting the importance to apply product content-based approaches. However, there is limited data available on chemical contents in products (not just PCPs) which are needed to estimate intakes (Egeghy et al., 2012); for example in this study, intakes were estimated for 325 of the chemicals that had chemical content data available, among the 518 PCP chemicals with estimated PiFs. Forthcoming efforts to estimate chemical contents in products will aid in increasing the amount of chemicals for which screening level exposures can be estimated (Egeghy et al., 2015).

We also demonstrated the use of the $\mathrm{PiF}$ and in vitro bioactivity data to estimate bioactive chemical contents. Comparing these values derived from the CPCPdb indicates that some users may be exposed to PCP chemicals at bioactive levels. In this study we only considered PCPs, however the Chemical and Product Categories (CPCat) database (Dionisio et al., 2015) indicates that all of these chemicals are also used in other product classes (e.g. household cleaners, building materials, etc.) such that exposure to PCPs may not represent all sources of exposure. Identifying all products in which a chemical occurs is needed to carry out chemical-specific aggregate exposure assessments and to identify bioactive chemical levels across product types and classes. 
The model presented here provides a simple analytical solution which can be used in HT exposure calculations and depends on physico-chemical properties which can be estimated for many chemicals using software such as EPISuite (U.S. EPA, 2012). The model, however, involves several assumptions. For example, we assumed that PCPs represent an aqueous solution and studied one chemical at a time, but other ingredients within a product could alter the skin permeability of the chemical from the product to the skin. We also used limited data to estimate the near-person inhalation during product use and further research may be needed to model this exposure pathway, especially for volatile chemicals. Several chemicals used in PCPs may have physico-chemical properties which cannot be readily estimated or applied to the $K_{p}^{a q}$ model, for example ionogenics and nanomaterials, both chemical classes which can be used in PCPs (Csiszar et al., 2011; Weir et al., 2012). As with all mass-balance based exposure models that take physico-chemical properties as input, there will be uncertainties associated with using these values. The PCP model in this paper depends on $\mathrm{K}_{\mathrm{aw}}$ and $\mathrm{K}_{\mathrm{ow}}$, which have uncertainties associated with them, especially when using estimated values. Figure 2a indicates, that for the 'leave-on' archetype (highest exposure scenario), the model is likely most sensitive to property values when there is competition between transfer to air and skin (diagonal on Figure 2a). The model will be less sensitive at the high- or low- ends of physico-chemical properties, for example, when $\log \mathrm{K}_{\mathrm{aw}}$ is small (e.g. $<-10) P i F^{u s e, t o t}$ approaches unity and is thus relatively insensitive to decreases in $\mathrm{K}_{\mathrm{aw}}$ (Figure 2a). Figure $2 \mathrm{~b}$ indicates that for wash-off products, $P$ F $^{\text {use,tot }}$ is more sensitive to physico-chemical properties as there is a more heterogeneous distribution of PiFuse,tot, due to the shorter exposure duration. For the chemicals in this study, 129 and 196 chemicals had both experimental and estimated $\log \mathrm{K}_{\mathrm{aw}}$ and $\log \mathrm{K}_{\mathrm{ow}}$ values, respectively, which on average 
differed by a factor of $\sim 1.5$ (experimental values were used in this study, when available). However, the discrepancy between measured and estimated properties was substantially larger for some chemicals, thus care should be taken when interpreting results when estimated properties are used.

Additionally, uncertainty can also occur based on which exposure pathways are included in the assessment. For example, we only included incidental ingestion for application to lips. For other leave-on products, this pathway may be important for chemicals with low volatility and skin permeability as these chemicals would remain on the skin surface longer and would thus be available for ingestion via, for example, hand-to-mouth behavior. Thus, in the context of prioritizing chemicals, it may be as important to screen not only for high exposure potentials, but also very low ones to make sure a pathway was not missed. The modeling framework presented here can be extended to include other exposure pathways. Recently, Fantke et al. (2016) proposed a matrix framework to calculate PiFs via several pathways designed with the flexibility to add pathways as needed or as more information becomes available about important exposure pathways. While calculations in this paper were deterministic, there can be considerable population variability in doses due to differences in factors such as individual PCP use and these calculations can also be done probabilistically to estimate population level exposure distributions (Csiszar et al., 2016). Uncertainty and variability in values can be taken into account for higher tier exposure estimates (Embry et al., 2014).

While the focus of this paper is to estimate human exposure to PCP chemicals, the use-stage PiF can also be used to inform chemical emission estimates to outdoor environments. For example, we also calculated the fraction of chemical emitted to urban air 
and to WWTPs and multiplying this by the mass of chemicals used in PCPs would yield outdoor emission estimates for these chemicals due to PCP use. Estimating environmental PCP chemical emissions has garnered interest as PCP chemicals have been detected in surface waters (Brausch and Rand, 2011; Gouin et al., 2013) and can potentially harm organisms and ecosystems. The percentage of applied chemical emitted to outdoor air and WWTPs both ranged from 0 to $\sim 100 \%$ and was highly dependent on the fractional amount of chemical that the individual user is exposed to. Considering environmental releases is also key when comparing human health impacts with ecological impacts such as those on aquatic ecosystems within broader chemical assessments.

\section{Acknowledgments}

We thank Kristin Isaacs (U.S. EPA) for the CPCPdb product category information. We thank Deborah Bennett (University of California, Davis) for helpful comments and discussion. Funding for the University of Michigan work was provided by U.S. EPA contract EP-14-C000115 on Development of Modular Risk Pathway Descriptions for Life Cycle Assessment, and the Long Range Research Initiative of the American Chemistry Council.

This research was supported in part by an appointment of S. Csiszar to the Postdoctoral Research Program at the National Risk Management Research Laboratory, Office of Research and Development, U.S. EPA administered by the Oak Ridge Institute for Science and Education through Interagency Agreement No. DW-89-92298301 between the U.S. Department of Energy and the U.S. Environmental Protection Agency.

The views expressed in this article are those of the authors and do not necessarily represent the views or policies of the U.S. Environmental Protection Agency.

\section{References}

Biesterbos, J.W., Dudzina, T., Delmaar, C.J., Bakker, M.I., Russel, F.G., von Goetz, N., Scheepers, P.T., Roeleveld, N., 2013. Usage patterns of personal care products: important factors for exposure assessment. Food Chem. Toxicol. 55, 8-17. 
Brausch, J.M., Rand, G.M., 2011. A review of personal care products in the aquatic environment: Environmental concentrations and toxicity. Chemosphere 82, 1518-1532.

CDC, 2009. Centers for Disease Control and Prevention, Fourth Report on Human Exposure to Environmental Chemicals. U.S. Department of Health and Human Services, Atlanta, Georgia.

Cohen Hubal, E.A., Richard, A., Aylward, L., Edwards, S., Gallagher, J., Goldsmith, M.R., Isukapalli, S., Tornero-Velez, R., Weber, E., Kavlock, R., 2010. Advancing exposure characterization for chemical evaluation and risk assessment. J Toxicol Environ Health B Crit Rev 13, 299-313.

Cowan-Ellsberry, C.E., Robison, S.H., 2009. Refining aggregate exposure: example using parabens. Regul Toxicol Pharmacol 55, 321-329.

Csiszar, S.A., Ernstoff, A.S., Fantke, P., Jolliet, O., 2016. Stochastic modeling of near-field exposure to parabens in personal care products. Journal of Exposure Science and Environmental Epidemiology. doi:10.1038/jes.2015.85.

Csiszar, S.A., Gandhi, N., Alexy, R., Benny, D.T., Struger, J., Marvin, C., Diamond, M.L., 2011. Aquivalence revisited - New model formulation and application to assess environmental fate of ionic pharmaceuticals in Hamilton Harbour, Lake Ontario. Environ. Int. 37, 821-828.

Dionisio, K.L., Frame, A.M., Goldsmith, M.-R., Wambaugh, J.F., Liddell, A., Cathey, T., Smith, D., Vail, J., Ernstoff, A.S., Fantke, P., Jolliet, O., Judson, R.S., 2015. Exploring consumer exposure pathways and patterns of use for chemicals in the environment. Toxicol. Rep. 2, 228-237.

Dudzina, T., Delmaar, C.J.E., Biesterbos, J.W.H., Bakker, M.I., Bokkers, B.G.H., Scheepers, P.T.J., van Engelen, J.G.M., Hungerbuehler, K., von Goetz, N., 2015. The probabilistic aggregate consumer exposure model (PACEM): Validation and comparison to a lower-tier assessment for the cyclic siloxane D5. Environ. Int. 79, 8-16.

Egeghy, P., R. Judson, S. Gangwal, S. Mosher, D. Smith, J. Vail, Hubal, E.A.C., 2012. The exposure data landscape for manufactured chemicals. Sci. Total Environ. 414, 159-166.

Egeghy, P.P., Sheldon, L.S., Isaacs, K.K., Halûk Özkaynak, Goldsmith, M.-R., Wambaugh, J.F., Judson, R.S., Buckley, T.J., 2015. Computational Exposure Science: An Emerging Discipline to Support 21st-Century Risk Assessment. Environ. Health Perspect.

Egeghy, P.P., Vallero, D.A., Cohen Hubal, E.A., 2011. Exposure-based prioritization of chemicals for risk assessment. Environ. Sci. Policy 14, 950-964.

Embry, M.R., Bachman, A.N., Bell, D.R., Boobis, A.R., Cohen, S.M., Dellarco, M., Dewhurst, I.C., Doerrer, N.G., Hines, R.N., Moretto, A., Pastoor, T.P., Phillips, R.D., Rowlands, J.C., Tanir, J.Y., Wolf, D.C., Doe, J.E., 2014. Risk assessment in the 21st century: roadmap and matrix. Crit. Rev. Toxicol. 44 Suppl 3, 6-16. 
Ernstoff, A.S., Fantke, P., Csiszar, S.A., Henderson, A.D., Chung, S., Jolliet, O., 2016. Multi-pathway exposure modelling of chemicals in cosmetics with application to shampoo. Environ. Int. 92-93, 87-86.

Fantke, P., Ernstoff, A.S., Huang, L., Csiszar, S.A., Jolliet, O., 2016. Coupled near-field and far-field exposure assessment framework for chemicals in consumer products. Environ. Int. http://dx.doi.org/10.1016/j.envint.2016.06.010.

Goldsmith, M.R., Grulke, C.M., Brooks, R.D., Transue, T.R., Tan, Y.M., Frame, A., Egeghy, P.P., Edwards, R., Chang, D.T., Tornero-Velez, R., Isaacs, K., Wang, A., Johnson, J., Holm, K., Reich, M., Mitchell, J., Vallero, D.A., Phillips, L., Phillips, M., Wambaugh, J.F., Judson, R.S., Buckley, T.J., Dary, C.C., 2014. Development of a consumer product ingredient database for chemical exposure screening and prioritization. Food Chem. Toxicol. 65, 269279.

Gouin, T., van Egmond, R., Sparham, C., Hastie, C., Chowdhury, N., 2013. Simulated use and wash-off release of decamethylcyclopentasiloxane used in anti-perspirants. Chemosphere 93, 726-734.

Harley, K., Kogut, K., Madrigal, D., Cardenas, M., Vera, I., Meza-Alfaro, G., She, J., Gavin, Q., Zahedi, R., Bradman, A., Eskenazi, B., Parra, K., 2016. Reducing Phthalate, Paraben, and Phenol Exposure from Personal Care Products in Adolescent Girls: Findings from the HERMOSA Intervention Study. Environ. Health Perspect. http://dx.doi.org/10.1289/ehp.1510514.

Hellweg, S., Demou, E., Bruzzi, R., Meijer, A., Rosenbaum, R., Huijbregts, M., McKone, T., 2009. Integrating Human Indoor Air Pollutant Exposure within Life Cycle Impact Assessment. Environ. Sci. Technol. 43, 1670-1679.

Isaacs, K.K., Glen, W.G., Egeghy, P., Goldsmith, M.R., Smith, L., Vallero, D., Brooks, R., Grulke, C.M., Özkaynak, H., 2014. SHEDS-HT: An Integrated Probabilistic Exposure Model for Prioritizing Exposures to Chemicals with Near-Field and Dietary Sources. Environ. Sci. Technol. 48, 12750-12759.

Jolliet, O., Ernstoff, A.S., Csiszar, S.A., Fantke, P., 2015. Defining Product Intake Fraction to Quantify and Compare Exposure to Consumer Products. Environ. Sci. Technol. 49, 89248931.

Kavlock, R., Chandler, K., Houck, K., Hunter, S., Judson, R., Kleinstreuer, N., Knudsen, T., Martin, M., Padilla, S., Reif, D., Richard, A., Rotroff, D., Sipes, N., Dix, D., 2012. Update on EPA's ToxCast program: providing high throughput decision support tools for chemical risk management. Chem. Res. Toxicol. 25, 1287-1302.

Loretz, L., Api, A.M., Barraj, L., Burdick, J., Davis de, A., Dressler, W., Gilberti, E., Jarrett, G., Mann, S., Laurie Pan, Y.H., Re, T., Renskers, K., Scrafford, C., Vater, S., 2006. Exposure data for personal care products: hairspray, spray perfume, liquid foundation, shampoo, body wash, and solid antiperspirant. Food Chem. Toxicol. 44, 2008-2018. 
Loretz, L.J., Api, A.M., Babcock, L., Barraj, L.M., Burdick, J., Cater, K.C., Jarrett, G., Mann, S., Pan, Y.H., Re, T.A., Renskers, K.J., Scrafford, C.G., 2008. Exposure data for cosmetic products: facial cleanser, hair conditioner, and eye shadow. Food Chem. Toxicol. 46, 1516-1524.

Loretz, L.J., Api, A.M., Barraj, L.M., Burdick, J., Dressler, W.E., Gettings, S.D., Han Hsu, H., Pan, Y.H., Re, T.A., Renskers, K.J., Rothenstein, A., Scrafford, C.G., Sewall, C., 2005. Exposure data for cosmetic products: lipstick, body lotion, and face cream. Food Chem. Toxicol. 43, 279-291.

Nazaroff, W., Weschler, C.J., Little, J.C., Hubal, E.A., 2012. Intake to production ratio: a measure of exposure intimacy for manufactured chemicals. Environ. Health Perspect. 120, 1678-1683.

Parlett, L.E., Calafat, A.M., Swan, S.H., 2013. Women's exposure to phthalates in relation to use of personal care products. J. Expo. Sci. Environ. Epidemiol. 23, 197-206.

Rosenbaum, R., Bachmann, T., Huijbregts, M., Jolliet, O., Juraske, R., Koehler, A., Larsen, H., MacLeod, M., Margni, M., McKone, T., Payet, J., Schuhmacher, M., van de Meent, D., Hauschild, M., 2008. USEtox - The UNEP-SETAC toxicity model: recommended characterisation factors for human toxicity and freshwater ecotoxicity. Int. J. Life Cycle Assess. 7, 532-546.

Sandanger, T.M., Huber, S., Moe, M.K., Braathen, T., Leknes, H., Lund, E., 2011. Plasma concentrations of parabens in postmenopausal women and self-reported use of personal care products: the NOWAC postgenome study. J. Expo. Sci. Environ. Epidemiol. 21, 595-600.

Shin, H.M., Ernstoff, A., Arnot, J.A., Wetmore, B.A., Csiszar, S.A., Fantke, P., Zhang, X.M., McKone, T.E., Jolliet, O., Bennett, D.H., 2015. Risk-Based High-Throughput Chemical Screening and Prioritization using Exposure Models and in Vitro Bioactivity Assays. Environ. Sci. Technol. 49, 6760-6771.

ten Berge, W., 2009. A simple dermal absorption model: derivation and application. Chemosphere 75, 1440-1445.

U.S. EPA, 2012. Estimation Programs Interface Suite ${ }^{\mathrm{TM}}$ for Microsoft ${ }^{\circledR}$ Windows, v 4.11 U.S. Environmental Protection Agency, Washington, DC, USA.

U.S. NIH, 2015. Household Products Database: Health \& Safety Information on Household Products. U.S. National Institutes of Health, Health \& Human Services, National Library of Medicine, Bethesda, MD.

Wambaugh, J., Setzer, R.W., Reif, D.M., Gangwal, S., Mitchell-Blackwood, J., Arnot, J.A., Jolliet, O., Frame, A., Rabinowitz, J., Knudsen, T.B., Judson, R.S., Egeghy, P., Vallero, D.A., Cohen Hubal, E.A., 2013. High-Throughput Models for Exposure-Based Chemical Prioritization in the ExpoCast Project. Environ. Sci. Technol. 47. 
Wambaugh, J.F., Wang, A., Dionisio, K.L., Frame, A., Egeghy, P., Judson, R., Setzer, R.W., 2014. High throughput heuristics for prioritizing human exposure to environmental chemicals. Environ. Sci. Technol. 48, 12760-12767.

Weir, A., Westerhoff, P., Fabricius, L., Hristovski, K., von Goetz, N., 2012. Titanium Dioxide Nanoparticles in Food and Personal Care Products. Environ. Sci. Technol. 46, 22422250.

Weschler, C.J., Nazaroff, W.W., 2014. Dermal Uptake of Organic Vapors Commonly Found in Indoor Air. Environ. Sci. Technol. 48, 1230-1237.

Wetmore, B.A., Wambaugh, J.F., Allen, B., Ferguson, S.S., Sochaski, M.A., Setzer, R.W., Houck, K.A., Strope, C.L., Cantwell, K., Judson, R.S., LeCluyse, E., Clewell, H.J., Thomas, R.S., Andersen, M.E., 2015. Incorporating High-Throughput Exposure Predictions With Dosimetry-Adjusted In Vitro Bioactivity to Inform Chemical Toxicity Testing. Toxicol. Sci. 148, 121-136.

Wetmore, B.A., Wambaugh;, J.F., Ferguson;, S.S., Sochaski;, M.A., Rotroff;, D.M., Freeman;, K., H.J. Clewell, I., Dix;, D.J., Andersen; M.E., Houck;, K.A., Allen;, B., Judson;, R.S., Singh;, R., Kavlock;, R.J., Richard;, A.M., Thomas, R.S., 2012. Integration of dosimetry, exposure, and high-throughput screening data in chemical toxicity assessment. Toxicol. Sci. 125, 157-174.

Wu, X.M., Bennett, D.H., Ritz, B., Cassady, D.L., Lee, K., Hertz-Picciotto, I., 2010. Usage pattern of personal care products in California households. Food Chem. Toxicol. 48, 31093119. 